P116 (continued)

Study Design, Setting, Participants, and Intervention: 2,477 students attending SNAP-Ed eligible schools in Alabama were randomly assigned to treatment $(n=1,674)$ and control $(n=803)$ groups. To assess daily F/V consumption, tally sheets customized with school lunch menus were distributed to students and collected weekly by SNAP-Ed educators. Students self-reported foods consumed on individual tally sheets immediately following lunch for each 5day period during the 17-class program. Between preand post-periods, treatment group students received weekly nutrition classes supplemented by weekly F/V tastings.

Outcome, Measures and Analysis: Growth modeling examined patterns of change in $\mathrm{F} / \mathrm{V}$ consumption at 17 time points.

Results: At post-period, there were significant increases in consumption of available school lunch fruit $(\mathrm{p}<.01)$ and vegetables $(\mathrm{p}<.001)$ in treatment group students compared to control group students. When observing only treatment group students, there were significant increases in fruit $(\mathrm{p}<.001)$ and vegetable $(\mathrm{p}<.001)$ consumptions from pre to post. Both F/V consumptions increased up to class 10 , and then stabilized.

Conclusions and Implications: $F / V$ intakes of youth can be increased through implementation of SNAP-Ed childhood obesity prevention programs. Long-term programs lasting at least 10 classes are desirable to allow students to adopt new F/V habits.

Funding: Supplemental Nutrition Assistance Program Education.

\section{P117 Rethinking Extension Communications: Are Factsheets Relevant in Today's Technology-based World?}

Laura Bellows,PhD, MPH, RD, laura.bellows@colostate. edu, Colorado State University, Department of Food Science \& Human Nutrition, 1571 Campus Delivery, Fort Collins, CO 80523-1571; C. Rosen; R. Moore;

E. Shackelton, MS

Objective: To understand how current Extension nutrition and health factsheets are utilized on the web and to illicit consumer input on preferred format and topics for online nutrition and health information.

Theory, Prior Research, Rationale: Eight-one percent of Americans use the internet -via computer or mobile device- and 59\% search for health information online. Many Extension websites provide nutrition information in the form of links to factsheets - educational materials originally designed for print and not the web.

Study Design, Setting, Participants, and Intervention: Google analytics were used to calculate website visitors, page views, and traffic sources. An online survey was developed and disseminated to adults, via a snowball recruitment method, to ascertain use of web and other technologies, perception of credible health information, nutrition and health topics of interest, and format that they would like nutrition information presented.

Outcome, Measures and Analysis: Google Analytics counts for visits, pageviews, time on page and bounce rates - were examined. Frequencies for survey results were calculated in SPSS by gender and age group.

Results: Fact sheets on nutrition topics received $1,708,512$ website pageviews (www.ext.colostate.edu) in 2012 making up 32\% of all Extension pageviews. The bounce rate was very high at $90.7 \%$. Survey respondents $(\mathrm{n}=427)$ indicated credibility as top factor in choosing a health website; $40 \%$ stated they were not familiar with or would never use Extension compared to $22 \%$ for universities.

Conclusions and Implications: Consumers want timely, unbiased, and research-based information online. Current factsheets are receiving a lot of pageviews, however, consumers are not staying long on the page. Extension and universities have an opportunity to repackage information into credible, consumer- and web-friendly format.

Funding: None.

\section{P118 Seasonal Differences in Outdoor Activity Time and Vitamin D Status of Young Korean Women}

Jin-Sook Yoon,PhD, jsook@kmu.ac.kr, Keimyung

University, 1000 Sindang-dong, Dalseo-gu, Daegu, South Korea; M. K. Song, MS, RD; Y. J. Cho, $M S, R D$

Objective: This study examines the seasonal differences in outdoor activity times and dietary vitamin D intakes, and explicates their relative impact on improving serum vitamin D status among Korean women.

Theory, Prior Research, Rationale: Vitamin D comes from diet as well as exposure to sunlight.

Study Design, Setting, Participants, and Intervention: This is a cross-sectional study of 258 freeliving women aged 20 to 30 years in Daegu, Korea. Dietary intake and daily physical activity patterns were collected for summer and winter.

Outcome, Measures and Analysis: We compared the results from 117 women for summer and 151 women for winter. Dietary intake of vitamin D was assessed by 24 hour recall method. Daily outdoor activity times were derived from physical activity diary. Serum 25$\mathrm{OH}$-vitamin D status was measured from fasting blood.

Results: The average dietary intake of vitamin D of the participants was $2.1 \mu \mathrm{g}$ during summer, $4.1 \mu \mathrm{g}$ during winter, showing no significant difference between the two seasons. Times spent on outdoor activities $(\mathrm{p}<0.01)$ in the summer $(=57.6 \pm 31.6 \mathrm{~min})$ were much longer than that in the winter $(=40.7 \pm 35.6 \mathrm{~min})$. The serum 25- $(\mathrm{OH})$ vitamin $\mathrm{D}$ levels of participants was $18.0 \pm 8.3 \mathrm{ng} / \mathrm{mL}$ in the summer and $13.3 \pm 4.3 \mathrm{ng} / \mathrm{mL}$ in the winter, showing that the latter was significantly lower than that of the former $(\mathrm{p}<0.001)$.

Continued on page 547 